\title{
The Metric Dimension of Some Generalized Petersen Graphs
}

\author{
Zehui Shao $\mathbb{D}^{1},{ }^{1}$ S. M. Sheikholeslami, ${ }^{2}$ Pu Wu, ${ }^{1,3}$ and Jia-Biao Liu $\mathbb{D}^{4}$ \\ ${ }^{1}$ Institute of Computing Science and Technology, Guangzhou University, Guangzhou 510006, China \\ ${ }^{2}$ Department of Mathematics, Azarbaijan Shahid Madani University, Tabriz, Iran \\ ${ }^{3}$ School of Information Science and Engineering, Chengdu University, Chengdu 610106, China \\ ${ }^{4}$ School of Mathematics and Physics, Anhui Jianzhu University, Hefei 230601, China \\ Correspondence should be addressed to Jia-Biao Liu; liujiabaoad@163.com
}

Received 26 March 2018; Accepted 9 July 2018; Published 1 August 2018

Academic Editor: Xiaohua Ding

Copyright (c) 2018 Zehui Shao et al. This is an open access article distributed under the Creative Commons Attribution License, which permits unrestricted use, distribution, and reproduction in any medium, provided the original work is properly cited.

The distance $d(u, v)$ between two distinct vertices $u$ and $v$ in a graph $G$ is the length of a shortest $(u, v)$-path in $G$. For an ordered subset $W=\left\{w_{1}, w_{2}, \ldots, w_{k}\right\}$ of vertices and a vertex $v$ in $G$, the code of $v$ with respect to $W$ is the ordered $k$-tuple $c_{W}(v)=\left(d\left(v, w_{1}\right), d\left(v, w_{2}\right), \ldots, d\left(v, w_{k}\right)\right)$. The set $W$ is a resolving set for $G$ if every two vertices of $G$ have distinct codes. The metric dimension of $G$ is the minimum cardinality of a resolving set of $G$. In this paper, we first extend the results of the metric dimension of $P(n, 3)$ and $P(n, 4)$ and study bounds on the metric dimension of the families of the generalized Petersen graphs $P(2 k, k)$ and $P(3 k, k)$. The obtained results mean that these families of graphs have constant metric dimension.

\section{Introduction}

Let $G$ be a connected graph with vertex set $V=V(G)$ and edge set $E=E(G)$. The distance between two distinct vertices $u$ and $v$ in $G$, denoted by $d(u, v)$, is the length of a shortest $(u, v)$-path. For positive integer $k$ and a vertex $v \in V(G)$, the $k$-neighborhood of $v$ is the set $N_{k}(v)=\{u \mid d(u, v)=k\}$. For an ordered subset $W=\left\{w_{1}, w_{2}, \ldots, w_{k}\right\}$ of vertices and a vertex $v$ in $G$, the code of $v$ with respect to $W$ is the ordered $k$ tuple $c_{W}(v)=\left(d\left(v, w_{1}\right), d\left(v, w_{2}\right), \ldots, d\left(v, w_{k}\right)\right)$. The set $W$ is a resolving set [1] (or locating set [2]) for $G$ if every two vertices of $G$ have distinct codes. The metric dimension of $G$, denoted by $\operatorname{dim}(G)$, is the minimum cardinality of a resolving set of $G$. A resolving set containing a minimum number of vertices is called a basis for $G$ [3].

Graph theory is a powerful tool to model the real world applications such as physical-chemical property testing [4, 5]. Motivated by the problem of uniquely determining the location of an intruder in a network, the concept of metric dimension of a graph was introduced by Slater in [6], where the metric generators were called locating sets. The concept of metric dimension of a graph was also introduced by Harary and Melter in [1], where metric generators were called resolving sets. Applications of this invariant to the navigation of robots in networks are discussed in [7] and applications to chemistry in $[8,9]$. This graph parameter was studied further in a number of other papers including, for instance, [10-19]. Several variations of metric generators including resolving dominating sets [20], independent resolving sets [21], local metric sets [22], strong resolving sets [23], mixed metric dimension [24], and $k$-metric dimension [25] have since been introduced and studied.

We observe from definition that the property of a given set $W$ of vertices of a graph $G$ to be a resolving set of $G$ can be tested by investigating the vertices of $V(G) \backslash W$ because every vertex $w \in W$ is the unique vertex of $G$ whose distance from $w$ is 0 . If $d(x, t) \neq d(y, t)$, we say that vertex $t$ distinguishes vertices $x$ and $y$.

For natural numbers $n$ and $k$, where $n>2 k$, a generalized Petersen graph $P(n, k)$ is a graph with vertex set $U \cup V$, where $U=\left\{u_{1}, u_{2}, \ldots, u_{n}\right\}$ and $V=\left\{v_{1}, v_{2}, \ldots, v_{n}\right\}$, and edge set $E_{1} \cup E_{2} \cup E_{3}$, where $E_{1}=\left\{u_{i} u_{i+1} \mid 1 \leq i \leq n, E_{2}=\left\{u_{i} v_{i} \mid\right.\right.$ $1 \leq i \leq n\}$, and $E_{3}=\left\{v_{i} v_{i+k} \mid 1 \leq i \leq n\right\}$, where subscripts are taken modulo $n$ (see $[2,26])$. We observe that, for each $1 \leq i \leq n$,

$$
\begin{aligned}
& N_{2}\left(u_{i}\right)=\left\{u_{i-2}, u_{i+2}, v_{i-1}, v_{i+1}, v_{i-k}, v_{i+k}\right\}, \\
& N_{2}\left(v_{i}\right)=\left\{u_{i-1}, u_{i+1}, v_{i-2 k}, v_{i+2 k}, u_{i-k}, u_{i+k}\right\},
\end{aligned}
$$


TABLE 1: The code $c_{W}(v)$ of $v$ with respect to $W=\left\{u_{1}, u_{7}, v_{5}\right\}$ in $P(9,3)$.

\begin{tabular}{cccccccccc}
\hline$v$ & $c_{W}(v)$ & $v$ & $c_{W}(v)$ & $v$ & $c_{W}(v)$ & $v$ & $c_{W}(v)$ & $v$ & $c_{W}(v)$ \\
\hline$u_{1}$ & $(0,3,3)$ & $u_{2}$ & $(1,4,2)$ & $u_{3}$ & $(2,4,3)$ & $u_{4}$ & $(3,3,2)$ & $u_{5}$ & $(4,2,1)$ \\
$u_{6}$ & $(4,1,2)$ & $u_{7}$ & $(3,0,3)$ & $u_{8}$ & $(2,1,2)$ & $u_{9}$ & $(1,2,3)$ & $v_{1}$ & $(1,2,4)$ \\
$v_{2}$ & $(2,3,1)$ & $v_{3}$ & $(3,3,4)$ & $v_{4}$ & $(2,2,3)$ & $v_{5}$ & $(3,3,0)$ & $v_{6}$ & $(3,2,3)$ \\
$v_{7}$ & $(2,1,4)$ & $v_{8}$ & $(3,2,1)$ & $v_{9}$ & $(2,3,4)$ & & & & \\
\hline
\end{tabular}

$$
\begin{aligned}
& N_{3}\left(u_{i}\right)=\left\{u_{i-3}, u_{i+3}, v_{i-2}, v_{i+2}, v_{i+1-k}, v_{i+k+1}, v_{i-1-k}, v_{i+k-1},\right. \\
& \left.\quad v_{i-2 k}, v_{i+2 k}, u_{i-k}, u_{i+k}\right\} \\
& N_{3}\left(v_{i}\right)=\left\{u_{i-2}, u_{i+2}, v_{i+1}, v_{i-1}, u_{i+1+k}, u_{i+k-1}, u_{i-1-k},\right. \\
& \left.\quad u_{i-k+1}, u_{i-2 k}, u_{i+2 k}, v_{i-3 k}, v_{i+3 k}\right\} .
\end{aligned}
$$

If $k=3$ and $n \geq 16$, then clearly $\left|N_{2}(x)\right| \geq 6$ and $\left|N_{3}(x)\right| \geq 8$ for each $x \in V(P(n, k))$.

Javaid et al. [27] proved that $\operatorname{dim}(P(n, 2))=3$ for $n \geq 5$ and posed the following problem.

Problem 1. Is the generalized Petersen graphs $P(n, k)$, for $n \geq$ 7 and $3 \leq k \leq\lfloor(n-1) / 2\rfloor$, a family of graphs with constant metric dimension?

Some partial answers are given to aforementioned problem as follows.

Theorem 2 (see [28]). For $n \geq 3, \operatorname{dim}(P(n, 1))=2$ when $n \equiv$ $1(\bmod 2)$ and $\operatorname{dim}(P(n, 1))=3$ if $n \equiv 0(\bmod 2)$.

Theorem 3 (see [29]).

$$
\operatorname{dim}(P(n, 3)) \begin{cases}=4, & \text { if } n \equiv 0(\bmod 6) \text { and } n \geq 24 \\ =3, & \text { if } n \equiv 1(\bmod 6) \text { and } n \geq 25 \\ \leq 5, & \text { if } n \equiv 2(\bmod 6) \text { and } n \geq 8 \\ \leq 4, & \text { if } n \equiv 3,4,5(\bmod 6) \text { and } n \geq 17\end{cases}
$$

In $[30,31]$, it was showed that

Theorem 4. (i) $\operatorname{dim}(P(2 k+1, k))=3$ if $k \geq 2$.

(ii)

$\operatorname{dim}(P(2 k, k-1))= \begin{cases}3, & \text { if } k=2 \text { or } k \geq 3 \text { is odd } \\ 4, & \text { if } k \geq 4 \text { is odd }\end{cases}$

In [32], it was proved that

Theorem 5. For $n \geq 4, \operatorname{dim}(P(n, 4))=3$ if $n \equiv 0(\bmod 4)$, $\operatorname{dim}(P(n, 4))=4$ when $n=4 k+3$ for even $k$, and $\operatorname{dim}(P(n, 4)) \leq 4$ otherwise. 5 .

In this paper, we first extend the results of Theorems 3 and

We make use of the following result in this paper.

Theorem 6 (see [7]). If $G$ is a graph of order $n$, diameter $d$ and metric dimension $k$, then $n<d^{k}+k$.

\section{Main Results}

Next result extends Theorem 3.

Lemma 7. Let $G$ be a connected graph and let $\left|N_{2}(v)\right| \geq 6$ or $\left|N_{3}(v)\right| \geq 8$ for each $v \in V(G)$. Then $\operatorname{dim}(G) \geq 3$.

Proof. Clearly, for any $w, v \in V(G)$ and for any $z \in N_{k}(w)$ we have

$$
d(v, w)-k \leq d(v, z) \leq d(v, w)+k
$$

Suppose, to the contrary, that $S=\left\{w_{1}, w_{2}\right\}$ is a resolving set of $G$. Since $\left|N_{2}\left(w_{1}\right)\right| \geq 6$ or $\left|N_{3}\left(w_{1}\right)\right| \geq 8$, we deduce from (7) and the Pigeonhole principle that there exist two vertices $x_{1}, x_{2} \in N_{2}\left(w_{1}\right)$ such that $d\left(x_{1}, w_{2}\right)=d\left(x_{2}, w_{2}\right)$, a contradiction.

Theorem 8. For $n \geq 7$,

(i) If $n \in\{9,10,11,15\}$ or $n \equiv 1(\bmod 6)$, then $\operatorname{dim}(P(n, 3))=3$.

(ii) If $n=20$, then $\operatorname{dim}(P(n, 3))=5$.

Proof. If $n=9$, then let $W=\left\{u_{1}, u_{7}, v_{5}\right\}$. The code of $v$ with respect to $W$ in $P(9,3)$ is presented in Table 1 yielding $\operatorname{dim}(P(9,3)) \leq 3$.

Now, we show that $\operatorname{dim}(P(9,3)) \geq 3$. Suppose, to the contrary, there exists a resolving set $W=\{x, y\}$ of $P(9,3)$. First let $W \cap U \neq \emptyset$. We may assume w.l.o.g. that $x \in$ $W \cap U$. By (1), we have $\left|N_{2}(x)\right|=6$. For each $u \in N_{2}(x)$, we have $d(y, x)-2 \leq d(y, u) \leq d(y, x)+2$. By the Pigeonhole principle, we have $d(y, u)=d(y, v)$ for some $u, v \in N_{2}(x)$ and this leads to a contradiction. Now let $W \cap U=\emptyset$. Assume without loss of generality that $x=v_{1}$ and $y=v_{i}$ for some $i \in\{2,3,4,5\}$. If $i \in\{2,3\}$, then $\left(d\left(x, u_{4}\right), d\left(y, u_{4}\right)\right)=\left(d\left(x, u_{9}\right), d\left(y, u_{9}\right)\right)$, and if $i \in\{4,5\}$, then $\left(d\left(x, u_{7-i}\right), d\left(y, u_{7-i}\right)\right)=\left(d\left(x, u_{9-i}\right),\left(y, u_{9-i}\right)\right)$, a contradiction. Thus, $\operatorname{dim}(P(9,3)) \geq 3$ and so $\operatorname{dim}(P(9,3))=3$.

If $n=10$, then let $W=\left\{u_{1}, v_{9}, v_{10}\right\}$. The code of $v$ with respect to $W$ in $P(10,3)$ is presented in Table 2 showing that $\operatorname{dim}(P(10,3)) \leq 3$.

Next, we show that $\operatorname{dim}(P(10,3)) \geq 3$. Suppose, to the contrary, there exists a resolving set $W=\{x, y\}$ of $P(10,3)$. As above, we may assume that $W \cap U=\emptyset$. We may assume w.l.o.g. that $x=v_{1}$ and $y=v_{i}$ for some $i \in\{2,3, \ldots, 6\}$. If $i \in\{2,4,6\}$, then $\left(d\left(x, u_{3}\right), d\left(y, u_{3}\right)\right)=\left(d\left(x, u_{5}\right), d\left(y, u_{5}\right)\right)$, and if $i \in\{3,5\}$, then we have $\left(d\left(x, u_{2}\right), d\left(y, u_{2}\right)\right)=\left(d\left(x, u_{4}\right)\right.$, $\left.d\left(y, u_{4}\right)\right)$, a contradiction.

If $n=11$, then let $W=\left\{u_{1}, u_{5}, v_{2}\right\}$. The code of $v$ with respect to $W$ in $P(11,3)$ is presented in Table 3 yielding $\operatorname{dim}(P(11,3)) \leq 3$. 
TABLE 2: The $\operatorname{code} c_{W}(v)$ of $v$ with respect to $W=\left\{u_{1}, v_{9}, v_{10}\right\}$ in $P(10,3)$.

\begin{tabular}{cccccccccc}
\hline$v$ & $c_{W}(v)$ & $v$ & $c_{W}(v)$ & $v$ & $c_{W}(v)$ & $v$ & $c_{W}(v)$ & $v$ & $c_{W}(v)$ \\
\hline$u_{1}$ & $(0,3,2)$ & $u_{2}$ & $(1,2,3)$ & $u_{3}$ & $(2,3,2)$ & $u_{4}$ & $(3,4,3)$ & $u_{5}$ & $(4,3,4)$ \\
$u_{6}$ & $(5,2,3)$ & $u_{7}$ & $(4,3,2)$ & $u_{8}$ & $(3,2,3)$ & $u_{9}$ & $(2,1,2)$ & $u_{10}$ & $(1,2,1)$ \\
$v_{1}$ & $(1,4,3)$ & $v_{2}$ & $(2,1,4)$ & $v_{3}$ & $(3,2,1)$ & $v_{4}$ & $(2,5,2)$ & $v_{5}$ & $(3,2,5)$ \\
$v_{6}$ & $(4,1,2)$ & $v_{7}$ & $(3,4,1)$ & $v_{8}$ & $(2,3,4)$ & $v_{9}$ & $(3,0,3)$ & $v_{10}$ & $(2,3,0)$ \\
\hline
\end{tabular}

TABLE 3: The code $c_{W}(v)$ of $v$ with respect to $W=\left\{u_{1}, u_{5}, v_{2}\right\}$ in $P(11,3)$.

\begin{tabular}{lccccccccc}
\hline$v$ & $c_{W}(v)$ & $v$ & $c_{W}(v)$ & $v$ & $c_{W}(v)$ & $v$ & $c_{W}(v)$ & $v$ & $c_{W}(v)$ \\
\hline$u_{1}$ & $(0,4,2)$ & $u_{2}$ & $(1,3,1)$ & $u_{3}$ & $(2,2,2)$ & $u_{4}$ & $(3,1,3)$ & $u_{5}$ & $(4,0,2)$ \\
$u_{6}$ & $(4,1,3)$ & $u_{7}$ & $(4,2,3)$ & $u_{8}$ & $(4,3,3)$ & $u_{9}$ & $(3,4,3)$ & $u_{10}$ & $(2,4,2)$ \\
$u_{11}$ & $(1,4,3)$ & $v_{1}$ & $(1,3,3)$ & $v_{2}$ & $(2,2,0)$ & $v_{3}$ & $(3,3,3)$ & $v_{4}$ & $(2,2,3)$ \\
$v_{5}$ & $(3,1,1)$ & $v_{6}$ & $(3,2,4)$ & $v_{7}$ & $(3,3,2)$ & $v_{8}$ & $(3,2,2)$ & $v_{9}$ & $(2,3,4)$ \\
$v_{10}$ & $(3,3,1)$ & $v_{11}$ & $(2,3,3)$ & & & & & \\
\hline
\end{tabular}

TABLE 4: The $\operatorname{code} c_{W}(v)$ of $v$ with respect to $W=\left\{u_{1}, u_{3}, v_{11}\right\}$ in $P(15,3)$.

\begin{tabular}{lccccccccc}
\hline$v$ & $c_{W}(v)$ & $v$ & $c_{W}(v)$ & $v$ & $c_{W}(v)$ & $v$ & $c_{W}(v)$ & $v$ & $c_{W}(v)$ \\
\hline$u_{1}$ & $(0,2,4)$ & $u_{2}$ & $(1,1,3)$ & $u_{3}$ & $(2,0,4)$ & $u_{4}$ & $(3,1,4)$ & $u_{5}$ & $(4,2,3)$ \\
$u_{6}$ & $(5,3,4)$ & $u_{7}$ & $(4,4,3)$ & $u_{8}$ & $(5,5,2)$ & $u_{9}$ & $(5,4,3)$ & $u_{10}$ & $(4,5,2)$ \\
$u_{11}$ & $(5,5,1)$ & $u_{12}$ & $(4,4,2)$ & $u_{13}$ & $(3,5,3)$ & $u_{14}$ & $(2,4,2)$ & $u_{15}$ & $(1,3,3)$ \\
$v_{1}$ & $(1,3,5)$ & $v_{2}$ & $(2,2,2)$ & $v_{3}$ & $(3,1,5)$ & $v_{4}$ & $(2,2,5)$ & $v_{5}$ & $(3,3,2)$ \\
$v_{6}$ & $(4,2,5)$ & $v_{7}$ & $(3,3,4)$ & $v_{8}$ & $(4,4,1)$ & $v_{9}$ & $(4,3,4)$ & $v_{10}$ & $(3,4,3)$ \\
$v_{11}$ & $(4,4,0)$ & $v_{12}$ & $(3,3,3)$ & $v_{13}$ & $(2,4,4)$ & $v_{14}$ & $(3,3,1)$ & $v_{15}$ & $(2,2,4)$ \\
\hline
\end{tabular}

TABLE 5: The code $c_{W}(v)$ of $v$ with respect to $W=\left\{u_{1}, u_{3}, u_{11}, v_{8}, v_{10}\right\}$ in $P(20,3)$.

\begin{tabular}{lccccccc}
\hline$v$ & $c_{W}(v)$ & $v$ & $c_{W}(v)$ & $v$ & $c_{W}(v)$ & $v$ & $c_{W}(v)$ \\
\hline$u_{1}$ & $(0,2,6,4,4)$ & $u_{2}$ & $(1,1,5,3,5)$ & $u_{3}$ & $(2,0,6,4,4)$ & $u_{4}$ & $(3,1,5,3,3)$ \\
$u_{5}$ & $(4,2,4,2,4)$ & $u_{6}$ & $(5,3,5,3,3)$ & $u_{7}$ & $(4,4,4,2,2)$ & $u_{8}$ & $(5,5,3,1,3)$ \\
$u_{9}$ & $(6,4,2,2,2)$ & $u_{10}$ & $(5,5,1,3,1)$ & $u_{11}$ & $(6,6,0,2,2)$ & $u_{12}$ & $(5,5,1,3,3)$ \\
$u_{13}$ & $(6,6,2,4,2)$ & $u_{14}$ & $(5,5,3,3,3)$ & $u_{15}$ & $(4,6,4,4,4)$ & $u_{16}$ & $(5,5,5,5,3)$ \\
$u_{17}$ & $(4,4,4,4,4)$ & $u_{18}$ & $(3,5,5,5,5)$ & $u_{19}$ & $(2,4,6,4,4)$ & $u_{20}$ & $(1,3,5,5,5)$ \\
$v_{1}$ & $(1,3,5,5,3)$ & $v_{2}$ & $(2,2,4,2,4)$ & $v_{3}$ & $(3,1,5,5,5)$ & $v_{4}$ & $(2,2,4,4,2)$ \\
$v_{5}$ & $(3,3,3,1,5)$ & $v_{6}$ & $(4,2,4,4,4)$ & $v_{7}$ & $(3,3,3,3,1)$ & $v_{8}$ & $(4,4,2,0,4)$ \\
$v_{9}$ & $(5,3,3,3,3)$ & $v_{10}$ & $(4,4,2,4,0)$ & $v_{11}$ & $(5,5,1,1,3)$ & $v_{12}$ & $(4,4,2,4,4)$ \\
$v_{13}$ & $(5,5,3,5,1)$ & $v_{14}$ & $(4,4,2,2,4)$ & $v_{15}$ & $(3,5,3,5,5)$ & $v_{16}$ & $(4,4,4,4,2)$ \\
$v_{17}$ & $(3,3,3,3,5)$ & $v_{18}$ & $(2,4,4,6,4)$ & $v_{19}$ & $(3,3,5,3,3)$ & $v_{20}$ & $(2,2,4,4,6)$ \\
\hline
\end{tabular}

Since $\operatorname{diam}(P(11,3))=4$, we deduce from Theorem 6 that $\operatorname{dim}(P(11,3)) \geq 3$. Thus, $\operatorname{dim}(P(11,3))=3$.

If $n=15$, then let $W=\left\{u_{1}, u_{3}, v_{11}\right\}$. The code of $v$ with respect to $W$ in $P(15,3)$ is presented in Table 4 implying that $\operatorname{dim}(P(15,3)) \leq 3$.

Since $\operatorname{diam}(P(15,3))=5$, it follows from Theorem 6 that $\operatorname{dim}(P(15,3)) \geq 3$. Hence, $\operatorname{dim}(P(15,3))=3$.

If $n=20$, then let $W=\left\{u_{1}, u_{3}, u_{11}, v_{8}, v_{10}\right\}$. The code of $v$ with respect to $W$ in $P(20,3)$ is presented in Table 5. This implies that $\operatorname{dim}(P(20,3)) \leq 5$.

Analogous to the proof of the case $n=9$, we can obtain the desired lower bound with a more complicated analysis. Also it can be verified by computer search.

If $n \leq 15$, we can verify the results by computer. If $n \geq 16$ and $n \equiv 1(\bmod 6)$, we have $\left|N_{2}(v)\right|=6$ and $\left|N_{3}(v)\right| \geq 8$ for any $v \in P(n, 3)$. Now by Lemma 7 , we have $\operatorname{dim}(P(n, 3)) \geq 3$. Now, the proof is complete.

The following theorem extends the result of Theorem 5 .

Theorem 9. Let $G$ be the graph $G=P(n, 4)$ with $n \geq 9$; then if $n \in\{9,10,11,13,17,21\}$ or $n \equiv 0(\bmod 4)$, then $\operatorname{dim}(G)=3$.

Proof. If $n=9$, let $W=\left\{u_{1}, v_{8}, v_{9}\right\}$. The code of $v$ with respect to $W$ in $P(9,4)$ is presented in Table 6 showing that $\operatorname{dim}(P(9,4)) \leq 3$.

If $n=10$, let $W=\left\{u_{1}, u_{5}, v_{3}\right\}$. The code of $v$ with respect to $W$ in $P(10,4)$ is presented in Table 7 showing that $\operatorname{dim}(P(10,4)) \leq 3$.

Note that the diameter of $P(10,4)$ is 4 ; by Theorem 6 , we have $\operatorname{dim}(P(10,4)) \geq 3$. 
TABLE 6: The code $c_{W}(v)$ of $v$ with respect to $W=\left\{u_{1}, v_{8}, v_{9}\right\}$ in $P(9,4)$.

\begin{tabular}{llllllllll}
\hline$v$ & $c_{W}(v)$ & $v$ & $c_{W}(v)$ & $v$ & $c_{W}(v)$ & $v$ & $c_{W}(v)$ & $v$ & $c_{W}(v)$ \\
\hline$u_{1}$ & $(0,3,2)$ & $u_{2}$ & $(1,3,3)$ & $u_{3}$ & $(2,2,3)$ & $u_{4}$ & $(3,2,2)$ & $u_{5}$ & $(3,3,2)$ \\
$u_{6}$ & $(3,3,3)$ & $u_{7}$ & $(3,2,3)$ & $u_{8}$ & $(2,1,2)$ & $u_{9}$ & $(1,2,1)$ & $v_{1}$ & $(1,4,2)$ \\
$v_{2}$ & $(2,3,4)$ & $v_{3}$ & $(3,1,3)$ & $v_{4}$ & $(3,1,1)$ & $v_{5}$ & $(2,3,1)$ & $v_{6}$ & $(2,4,3)$ \\
$v_{7}$ & $(3,2,4)$ & $v_{8}$ & $(3,0,2)$ & $v_{9}$ & $(2,2,0)$ & & & & \\
\hline
\end{tabular}

TABLE 7: The code $c_{W}(v)$ of $v$ with respect to $W=\left\{u_{1}, u_{5}, v_{3}\right\}$ in $P(10,4)$.

\begin{tabular}{lccccccccc}
\hline$v$ & $c_{W}(v)$ & $v$ & $c_{W}(v)$ & $v$ & $c_{W}(v)$ & $v$ & $c_{W}(v)$ & $v$ & $c_{W}(v)$ \\
\hline$u_{1}$ & $(0,3,3)$ & $u_{2}$ & $(1,3,2)$ & $u_{3}$ & $(2,2,1)$ & $u_{4}$ & $(3,1,2)$ & $u_{5}$ & $(3,0,3)$ \\
$u_{6}$ & $(4,1,3)$ & $u_{7}$ & $(3,2,2)$ & $u_{8}$ & $(3,3,3)$ & $u_{9}$ & $(2,3,2)$ & $u_{10}$ & $(1,4,3)$ \\
$v_{1}$ & $(1,2,2)$ & $v_{2}$ & $(2,3,3)$ & $v_{3}$ & $(3,3,0)$ & $v_{4}$ & $(3,2,3)$ & $v_{5}$ & $(2,1,2)$ \\
$v_{6}$ & $(3,2,4)$ & $v_{7}$ & $(2,3,1)$ & $v_{8}$ & $(3,3,4)$ & $v_{9}$ & $(3,2,1)$ & $v_{10}$ & $(2,3,4)$ \\
\hline
\end{tabular}

TABLE 8: The code $c_{W}(v)$ of $v$ with respect to $W=\left\{u_{1}, v_{8}, v_{11}\right\}$ in $P(11,4)$.

\begin{tabular}{llcccccccc}
\hline$v$ & $c_{W}(v)$ & $v$ & $c_{W}(v)$ & $v$ & $c_{W}(v)$ & $v$ & $c_{W}(v)$ & $v$ & $c_{W}(v)$ \\
\hline$u_{1}$ & $(0,2,2)$ & $u_{2}$ & $(1,3,3)$ & $u_{3}$ & $(2,3,3)$ & $u_{4}$ & $(3,2,2)$ & $u_{5}$ & $(3,3,3)$ \\
$u_{6}$ & $(4,3,3)$ & $u_{7}$ & $(4,2,2)$ & $u_{8}$ & $(3,1,3)$ & $u_{9}$ & $(3,2,3)$ & $u_{10}$ & $(2,3,2)$ \\
$u_{11}$ & $(1,3,1)$ & $v_{1}$ & $(1,1,3)$ & $v_{2}$ & $(2,4,4)$ & $v_{3}$ & $(3,4,2)$ & $v_{4}$ & $(3,1,1)$ \\
$v_{5}$ & $(2,2,4)$ & $v_{6}$ & $(3,4,4)$ & $v_{7}$ & $(3,3,1)$ & $v_{8}$ & $(2,0,2)$ & $v_{9}$ & $(3,3,4)$ \\
$v_{10}$ & $(3,4,3)$ & $v_{11}$ & $(2,2,0)$ & & & & & \\
\hline
\end{tabular}

TABLE 9: The $\operatorname{code} c_{W}(v)$ of $v$ with respect to $W=\left\{u_{1}, v_{14}, v_{17}\right\}$ in $P(17,4)$.

\begin{tabular}{lccccccccc}
\hline$v$ & $c_{W}(v)$ & $v$ & $c_{W}(v)$ & $v$ & $c_{W}(v)$ & $v$ & $c_{W}(v)$ & $v$ & $c_{W}(v)$ \\
\hline$u_{1}$ & $(0,2,2)$ & $u_{2}$ & $(1,3,3)$ & $u_{3}$ & $(2,4,3)$ & $u_{4}$ & $(3,4,2)$ & $u_{5}$ & $(3,3,3)$ \\
$u_{6}$ & $(4,3,4)$ & $u_{7}$ & $(5,4,4)$ & $u_{8}$ & $(5,4,3)$ & $u_{9}$ & $(4,3,3)$ & $u_{10}$ & $(4,2,4)$ \\
$u_{11}$ & $(5,3,4)$ & $u_{12}$ & $(5,3,3)$ & $u_{13}$ & $(4,2,2)$ & $u_{14}$ & $(3,1,3)$ & $u_{15}$ & $(3,2,3)$ \\
$u_{16}$ & $(2,3,2)$ & $u_{17}$ & $(1,3,1)$ & $v_{1}$ & $(1,1,3)$ & $v_{2}$ & $(2,3,4)$ & $v_{3}$ & $(3,5,4)$ \\
$v_{4}$ & $(3,5,1)$ & $v_{5}$ & $(2,2,3)$ & $v_{6}$ & $(3,2,5)$ & $v_{7}$ & $(4,5,5)$ & $v_{8}$ & $(4,5,2)$ \\
$v_{9}$ & $(3,3,2)$ & $v_{10}$ & $(3,1,5)$ & $v_{11}$ & $(4,4,5)$ & $v_{12}$ & $(4,4,3)$ & $v_{13}$ & $(3,3,1)$ \\
$v_{14}$ & $(2,0,4)$ & $v_{15}$ & $(3,3,4)$ & $v_{16}$ & $(3,4,3)$ & $v_{17}$ & $(2,4,0)$ & \\
\hline
\end{tabular}

If $n=11$, let $W=\left\{u_{1}, v_{8}, v_{11}\right\}$. The code of $v$ with respect to $W$ in $P(11,4)$ is presented in Table 8 showing that $\operatorname{dim}(P(11,4)) \leq 3$.

Note that the diameter of $P(11,4)$ is 4 ; by Theorem 6 , we have $\operatorname{dim}(P(11,4)) \geq 3$.

If $n=17$, let $W=\left\{u_{1}, v_{14}, v_{17}\right\}$. The code of $v$ with respect to $W$ in $P(17,4)$ is presented in Table 9 showing that $\operatorname{dim}(P(17,4)) \leq 3$.

Note that the diameter of $P(17,4)$ is 5 ; by Theorem 6 , we have $\operatorname{dim}(P(17,4)) \geq 3$.

If $n=21$, let $W=\left\{u_{1}, u_{16}, v_{14}\right\}$. The code of $v$ with respect to $W$ in $P(21,4)$ is presented in Table 10 showing that $\operatorname{dim}(P(21,4)) \leq 3$.

Note that the diameter of $P(21,4)$ is 6 ; by Theorem 6 , we have $\operatorname{dim}(P(21,4)) \geq 3$.

Theorem 10. Let $G$ be the graph $G=P(2 k, k)$ with $k \geq 3$; then

$$
\operatorname{dim}(G) \begin{cases}=3, & \text { if } k=3 \text { or } k \equiv 0(\bmod 2) \\ \leq 4, & \text { otherwise. }\end{cases}
$$

\section{Proof.}

Case $1(k=3$ or $k \equiv 0(\bmod 2))$. If $k=3$, let $W=\left\{u_{3}, v_{1}, v_{2}\right\}$. The code of $v$ with respect to $W$ in $P(6,3)$ is presented in Table 11 showing that $\operatorname{dim}(P(6,3)) \leq 3$.

If $k=4$, let $W=\left\{v_{4}, v_{7}, v_{8}\right\}$. The code of $v$ with respect to $W$ in $P(8,4)$ is presented in Table 12 showing that $\operatorname{dim}(P(8,4)) \leq 3$.

If $k \geq 6$, let $W=\left\{u_{1}, u_{2}, u_{k+1}\right\}$. Then the codes of the outer vertices are $c_{W}\left(u_{i}\right)=\left(h_{1}(i), h_{2}(i), h_{3}(i)\right)$ and the codes of the inner vertices are $c_{W}\left(v_{i}\right)=\left(g_{1}(i), g_{2}(i), g_{3}(i)\right)$, where

$$
h_{1}(i)= \begin{cases}i-1, & 3 \leq i \leq \frac{k}{2}+2 ; \\ k+4-i, & \frac{k}{2}+3 \leq i \leq k ; \\ i+2-k, & k+1 \leq i \leq \frac{3 k}{2}-1 ; \\ 2 k+1-i, & \frac{3 k}{2} \leq i \leq 2 k .\end{cases}
$$


TABLE 10: The code $c_{W}(v)$ of $v$ with respect to $W=\left\{u_{1}, u_{16}, v_{14}\right\}$ in $P(21,4)$.

\begin{tabular}{lccccccccc}
\hline$v$ & $c_{W}(v)$ & $v$ & $c_{W}(v)$ & $v$ & $c_{W}(v)$ & $v$ & $c_{W}(v)$ & $v$ & $c_{W}(v)$ \\
\hline$u_{1}$ & $(0,5,3)$ & $u_{2}$ & $(1,5,4)$ & $u_{3}$ & $(2,4,5)$ & $u_{4}$ & $(3,5,5)$ & $u_{5}$ & $(3,6,4)$ \\
$u_{6}$ & $(4,6,3)$ & $u_{7}$ & $(5,5,4)$ & $u_{8}$ & $(5,4,4)$ & $u_{9}$ & $(4,5,3)$ & $u_{10}$ & $(5,5,2)$ \\
$u_{11}$ & $(6,4,3)$ & $u_{12}$ & $(6,3,3)$ & $u_{13}$ & $(5,3,2)$ & $u_{14}$ & $(4,2,1)$ & $u_{15}$ & $(5,1,2)$ \\
$u_{16}$ & $(5,0,3)$ & $u_{17}$ & $(4,1,3)$ & $u_{18}$ & $(3,2,2)$ & $u_{19}$ & $(3,3,3)$ & $u_{20}$ & $(2,3,4)$ \\
$u_{21}$ & $(1,4,4)$ & $v_{1}$ & $(1,4,2)$ & $v_{2}$ & $(2,4,3)$ & $v_{3}$ & $(3,3,6)$ & $v_{4}$ & $(3,4,6)$ \\
$v_{5}$ & $(2,5,3)$ & $v_{6}$ & $(3,5,2)$ & $v_{7}$ & $(4,4,5)$ & $v_{8}$ & $(4,3,5)$ & $v_{9}$ & $(3,4,4)$ \\
$v_{10}$ & $(4,4,1)$ & $v_{11}$ & $(5,3,4)$ & $v_{12}$ & $(5,2,4)$ & $v_{13}$ & $(4,3,3)$ & $v_{14}$ & $(3,3,0)$ \\
$v_{15}$ & $(4,2,3)$ & $v_{16}$ & $(4,1,4)$ & $v_{17}$ & $(3,2,4)$ & $v_{18}$ & $(2,3,1)$ & $v_{19}$ & $(3,3,4)$ \\
$v_{20}$ & $(3,2,5)$ & $v_{21}$ & $(2,3,5)$ & & & & & & \\
\hline
\end{tabular}

TABLE 11: The $\operatorname{code} c_{W}(v)$ of $v$ with respect to $W=\left\{u_{3}, v_{1}, v_{2}\right\}$ in $P(6,3)$.

\begin{tabular}{lccccccccc}
\hline$v$ & $c_{W}(v)$ & $v$ & $c_{W}(v)$ & $v$ & $c_{W}(v)$ & $v$ & $c_{W}(v)$ & $v$ & $c_{W}(v)$ \\
\hline$u_{1}$ & $(2,1,2)$ & $u_{2}$ & $(1,2,1)$ & $u_{3}$ & $(0,3,2)$ & $u_{4}$ & $(1,2,3)$ & $u_{5}$ & $(2,3,2)$ \\
$u_{6}$ & $(3,2,3)$ & $v_{1}$ & $(3,0,3)$ & $v_{2}$ & $(2,3,0)$ & $v_{3}$ & $(1,4,3)$ & $v_{4}$ & $(2,1,4)$ \\
$v_{5}$ & $(3,4,1)$ & $v_{6}$ & $(2,3,4)$ & & & & & \\
\hline
\end{tabular}

TABLE 12: The code $c_{W}(v)$ of $v$ with respect to $W=\left\{v_{4}, v_{7}, v_{8}\right\}$ in $P(8,4)$.

\begin{tabular}{cccccccccc}
\hline$v$ & $c_{W}(v)$ & $v$ & $c_{W}(v)$ & $v$ & $c_{W}(v)$ & $v$ & $c_{W}(v)$ & $v$ & $c_{W}(v)$ \\
\hline$u_{1}$ & $(3,3,2)$ & $u_{2}$ & $(3,3,3)$ & $u_{3}$ & $(2,2,3)$ & $u_{4}$ & $(1,3,2)$ & $u_{5}$ & $(2,3,3)$ \\
$u_{6}$ & $(3,2,3)$ & $u_{7}$ & $(3,1,2)$ & $u_{8}$ & $(2,2,1)$ & $v_{1}$ & $(4,4,3)$ & $v_{2}$ & $(4,4,4)$ \\
$v_{3}$ & $(3,1,4)$ & $v_{4}$ & $(0,4,1)$ & $v_{5}$ & $(3,4,4)$ & $v_{6}$ & $(4,3,4)$ & $v_{7}$ & $(4,0,3)$ \\
$v_{8}$ & $(1,3,0)$ & & & & & & & & \\
\hline
\end{tabular}

$$
\begin{aligned}
& h_{2}(i)= \begin{cases}i-2, & 3 \leq i \leq \frac{k}{2}+3 ; \\
k+5-i, & \frac{k}{2}+4 \leq i \leq k+2 ; \\
i+1-k, & k+3 \leq i \leq \frac{3 k}{2} ; \\
2 k+2-i, & \frac{3 k}{2}+1 \leq i \leq 2 k .\end{cases} \\
& h_{3}(i)= \begin{cases}i+2, & 3 \leq i \leq \frac{k}{2}-1 ; \\
k+1-i, & \frac{k}{2} \leq i \leq k ; \\
i-1-k, & k+1 \leq i \leq \frac{3 k}{2}+2 ; \\
2 k+4-i, & \frac{3 k}{2}+3 \leq i \leq 2 k .\end{cases} \\
& g_{1}(i)= \begin{cases}i, & 1 \leq i \leq \frac{k}{2}+1 ; \\
i+1-k, & k+2 \leq i \leq \frac{3 k}{2} ; \\
2 k+2-i, & \frac{3 k}{2} \leq i \leq 2 k .\end{cases}
\end{aligned}
$$

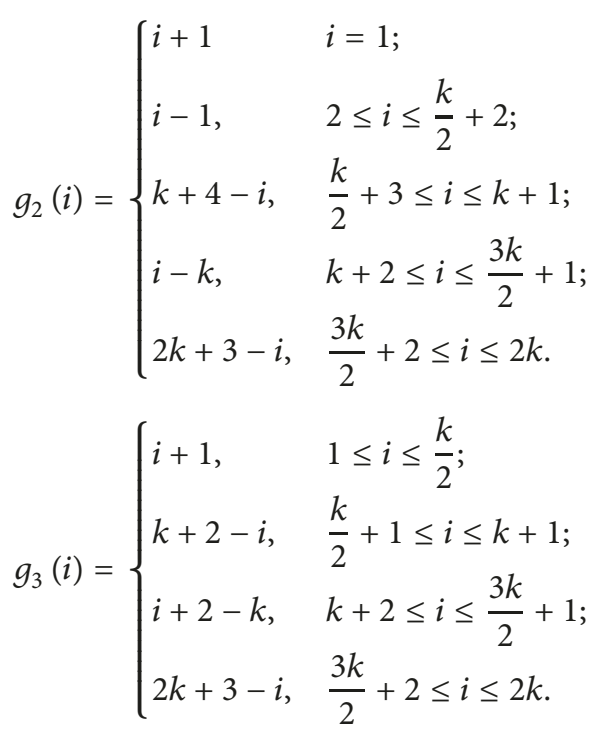

It can be verified that there are no two vertices on the outer cycle with the same codes, and there are no two vertices in the inner cycle and outer cycle with the same codes. Moreover, no two vertices in the inner cycle have same codes. Hence, $W=\left\{u_{1}, u_{2}, u_{k+1}\right\}$ is a resolving set of $P(2 k, k)$ for even $k \geq 6$. This means that $\operatorname{dim}(P(2 k, k)) \leq 3$ for even $k \geq 6$. 
TABLE 13: The code $c_{W}(v)$ of $v$ with respect to $W=\left\{u_{1}, v_{10}, v_{11}\right\}$ in $P(21,7)$.

\begin{tabular}{lccccccccc}
\hline$v$ & $c_{W}(v)$ & $v$ & $c_{W}(v)$ & $v$ & $c_{W}(v)$ & $v$ & $c_{W}(v)$ & $v$ & $c_{W}(v)$ \\
\hline$u_{1}$ & $(0,3,3)$ & $u_{2}$ & $(1,2,3)$ & $u_{3}$ & $(2,3,2)$ & $u_{4}$ & $(3,4,3)$ & $u_{5}$ & $(3,3,4)$ \\
$u_{6}$ & $(4,2,3)$ & $u_{7}$ & $(5,3,2)$ & $u_{8}$ & $(4,3,3)$ & $u_{9}$ & $(3,2,3)$ & $u_{10}$ & $(3,1,2)$ \\
$u_{11}$ & $(2,2,1)$ & $u_{12}$ & $(1,3,2)$ & $v_{1}$ & $(1,4,4)$ & $v_{2}$ & $(2,1,4)$ & $v_{3}$ & $(3,4,1)$ \\
$v_{4}$ & $(3,5,4)$ & $v_{5}$ & $(2,4,5)$ & $v_{6}$ & $(3,1,4)$ & $v_{7}$ & $(4,4,1)$ & $v_{8}$ & $(3,4,4)$ \\
$v_{9}$ & $(2,3,4)$ & $v_{10}$ & $(3,0,3)$ & $v_{11}$ & $(3,3,0)$ & $v_{12}$ & $(2,4,3)$ & \\
\hline
\end{tabular}

Case $2(k \equiv 1(\bmod 2)$ and $k \geq 5)$. Let $W=\left\{u_{1}, u_{2}\right.$, $\left.u_{k+1}, u_{k+2}\right\}$. Then the codes of the outer vertices are $c_{W}\left(u_{i}\right)=$ $\left(h_{1}(i), h_{2}(i), h_{3}(i), h_{4}(i)\right)$, where

$$
\begin{aligned}
& h_{1}(i)= \begin{cases}i-1, & 1 \leq i \leq \frac{k+1}{2}+2 ; \\
k+4-i, & \frac{k+1}{2}+3 \leq i \leq k+1 \\
i+2-k, & k+2 \leq i \leq \frac{3 k-1}{2} \\
2 k+1-i, & \frac{3 k+1}{2} \leq i \leq 2 k .\end{cases} \\
& h_{2}(i)= \begin{cases}i & i=1 ; \\
i-2, & 1 \leq i \leq \frac{k+1}{2}+3 ; \\
k+5-i, & \frac{k+1}{2}+4 \leq i \leq k+2 ; \\
i+1-k, & k+3 \leq i \leq \frac{3 k-1}{2} ; \\
2 k+2-i, & \frac{3 k+1}{2}+1 \leq i \leq 2 k .\end{cases} \\
& h_{3}(i)= \begin{cases}i+2, & 1 \leq i \leq \frac{k-1}{2} ; \\
k+1-i, & \frac{k+1}{2} \leq i \leq k ; \\
i-1-k, & k+1 \leq i \leq \frac{3 k+1}{2}+2 ; \\
2 k+4-i, & \frac{3 k+1}{2}+3 \leq i \leq 2 k .\end{cases} \\
& h_{4}(i)= \begin{cases}i+3 & i=1 ; \\
i+1, & 2 \leq i \leq \frac{k+1}{2} ; \\
k+2-i, & \frac{k+3}{2} \leq i \leq k+1 ; \\
i-2-k, & k+2 \leq i \leq \frac{3 k+1}{2}+3 ; \\
2 k+5-i, & \frac{3 k+1}{2}+4 \leq i \leq 2 k .\end{cases}
\end{aligned}
$$

Then the codes of the inner vertices are $c_{W}\left(v_{i}\right)=\left(g_{1}(i)\right.$, $\left.g_{2}(i), g_{3}(i), g_{4}(i)\right)$, where

$$
g_{1}(i)= \begin{cases}i, & 1 \leq i \leq \frac{k+1}{2}+1 \\ k+3-i, & \frac{k+3}{2} \leq i \leq k \\ i+1-k, & k+1 \leq i \leq \frac{3 k+1}{2} \\ 2 k+2-i, & \frac{3 k+3}{2} \leq i \leq 2 k\end{cases}
$$

$$
\begin{aligned}
& g_{2}(i)= \begin{cases}i+1 & i=1 ; \\
i-1, & 2 \leq i \leq \frac{k+3}{2} ; \\
k+4-i, & \frac{k+5}{2} \leq i \leq k+1 ; \\
i-k, & k+2 \leq i \leq \frac{3 k+1}{2} \\
2 k+3-i, & \frac{3 k+3}{2} \leq i \leq 2 k .\end{cases} \\
& g_{3}(i)= \begin{cases}i+1, & 1 \leq i \leq \frac{k+1}{2} ; \\
k+2-i, & \frac{k+3}{2} \leq i \leq k ; \\
i-k, & k+1 \leq i \leq \frac{3 k+1}{2} \\
2 k+3-i, & \frac{3 k+3}{2} \leq i \leq 2 k .\end{cases} \\
& g_{4}(i)= \begin{cases}i+2 & i=1 ; \\
i, & 2 \leq i \leq \frac{k+1}{2} ; \\
k+3-i, & \frac{k+3}{2} \leq i \leq k+1 ; \\
i-1-k, & k+2 \leq i \leq \frac{3 k+3}{2} ; \\
2 k+4-i, & \frac{3 k+5}{2} \leq i \leq 2 k .\end{cases}
\end{aligned}
$$

It can be verified that there are no two vertices on the outer cycle with the same codes, and there are no two vertices in the inner cycle and outer cycle with the same codes. Moreover, no two vertices in the inner cycle have same codes. Hence, $W=\left\{u_{1}, u_{2}, u_{k+1}, u_{k+2}\right\}$ is a resolving set of $P(2 k, k)$ for odd $k \geq 5$. This means that $\operatorname{dim}(P(2 k, k)) \leq 4$ for odd $k \geq 5$.

Theorem 11. Let $G$ be the graph $G=P(3 k, k)$ with $k \geq 3$; then

$$
\operatorname{dim}(G)= \begin{cases}4, & \text { if } k=7 \\ 3, & \text { otherwise }\end{cases}
$$

Proof.

Case $1(k \equiv 0(\bmod 2))$. If $k=4$, let $W=\left\{u_{1}, v_{10}, v_{11}\right\}$. The code of $v$ with respect to $W$ in $P(12,4)$ is presented in Table 13 showing that $\operatorname{dim}(P(12,4)) \leq 3$.

If $k \geq 6$, let $W=\left\{u_{1}, v_{k / 2+2}, v_{k+1}\right\}$. Then the codes of the outer vertices are $c_{W}\left(u_{i}\right)=\left(h_{1}(i), h_{2}(i), h_{3}(i)\right)$ and the 
codes of the inner vertices are $c_{W}\left(v_{i}\right)=\left(g_{1}(i), g_{2}(i), g_{3}(i)\right)$, where

$$
\begin{aligned}
& h_{1}(i)= \begin{cases}i-1, & 1 \leq i \leq \frac{k}{2}+2 \\
k+4-i, & \frac{k}{2}+3 \leq i \leq k+1 \\
i+2-k, & k+2 \leq i \leq \frac{3 k}{2}+1 ; \\
2 k+4-i, & \frac{3 k}{2}+2 \leq i \leq 2 k+1 \\
i-2 k+2, & 2 k+2 \leq i \leq \frac{5 k}{2}-1 ; \\
3 k-i+1, & \frac{5 k}{2} \leq i \leq 3 k .\end{cases} \\
& g_{1}(i)= \begin{cases}i, & 1 \leq i \leq \frac{k}{2}+1 ; \\
k+3-i, & \frac{k}{2}+2 \leq i \leq k+1 ; \\
i+1-k, & k+2 \leq i \leq \frac{3 k}{2}+1 ; \\
2 k+3-i, & \frac{3 k}{2}+2 \leq i \leq 2 k+1 ; \\
i-2 k+1, & 2 k+2 \leq i \leq \frac{5 k}{2} ; \\
3 k-i+2, & \frac{5 k}{2}+1 \leq i \leq 3 k .\end{cases} \\
& h_{2}(i)= \begin{cases}\frac{k}{2}+1, & i=1 \\
k-i-2, & 2 \leq i \leq \frac{k}{2}+2 \\
i-k+4, & \frac{k}{2}+3 \leq i \leq k+2 \\
2 k-i-1, & k+3 \leq i \leq \frac{3 k}{2}+2 \\
i-2 k+5, & \frac{3 k}{2}+3 \leq i \leq 2 k+2 \\
3 k-i-1, & 2 k+3 \leq i \leq \frac{5 k}{2}+2 \\
i-3 k+5, & \frac{5 k}{2}+3 \leq i \leq 3 k\end{cases} \\
& g_{2}(i)= \begin{cases}\frac{k}{2}+2, & i=1 \\
0, & i=\frac{k}{2}+2 \\
1, & i \in\left\{\frac{3 k}{2}+2, \frac{5 k}{2}+2\right\} \\
k-i-1, & 2 \leq i \leq \frac{k}{2}+1 \\
i-k+5, & \frac{k}{2}+3 \leq i \leq k+2 \\
2 k-i, & k+3 \leq i \leq \frac{3 k}{2}+1 \\
i-2 k+6, & \frac{3 k}{2}+3 \leq i \leq 2 k+2 \\
3 k-i, & 2 k+3 \leq i \leq \frac{5 k}{2}+1 \\
i-3 k+6, & \frac{5 k}{2}+3 \leq i \leq 3 k .\end{cases}
\end{aligned}
$$

$$
h_{3}(i)= \begin{cases}i+1, & 1 \leq i \leq \frac{k}{2} ; \\ k+2-i, & \frac{k}{2}+1 \leq i \leq k+1 ; \\ i-k, & k+2 \leq i \leq \frac{3 k}{2}+1 ; \\ 2 k+3-i, & \frac{3 k}{2}+2 \leq i \leq 2 k+1 ; \\ i-2 k+1, & 2 k+2 \leq i \leq \frac{5 k}{2}+1 ; \\ 3 k-i+3, & \frac{5 k}{2}+2 \leq i \leq 3 k .\end{cases}
$$$$
g_{3}(i)= \begin{cases}0, & i=k+1 ; \\ 1, & i \in\{1,2 k+1\} ; \\ i+2, & 2 \leq i \leq \frac{k}{2} ; \\ k-i+3, & \frac{k}{2}+1 \leq i \leq k ; \\ i-k+1, & k+2 \leq i \leq \frac{3 k}{2}+1 ; \\ 2 k-i+4, & \frac{3 k}{2}+2 \leq i \leq 2 k ; \\ i-2 k+2, & 2 k+2 \leq i \leq \frac{5 k}{2}+1 ; \\ 3 k-i+4, & \frac{5 k}{2}+2 \leq i \leq 3 k .\end{cases}
$$

It can be verified that there are no two vertices on the outer cycle with the same codes, and there are no two vertices in the inner cycle and outer cycle with the same codes. Moreover, no two vertices in the inner cycle have same codes. Hence, $W=\left\{u_{1}, v_{k / 2+2}, v_{k+1}\right\}$ is a resolving set of $P(3 k, k)$ for even $k \geq 6$. This means that $\operatorname{dim}(P(3 k, k)) \leq 3$ for even $k \geq 6$.

Case $2(k \equiv 1(\bmod 2)$ and $k \geq 5)$. If $k=5$, let $W=$ $\left\{v_{4}, v_{10}, v_{13}\right\}$. The code of $v$ with respect to $W$ in $P(15,5)$ is presented in Table 14 showing that $\operatorname{dim}(P(15,5)) \leq 3$.

Note that the diameter of $P(15,5)$ is 5 ; by Theorem 6 , we have $\operatorname{dim}(P(15,5)) \geq 3$.

If $k=7$, we can confirm that $\operatorname{dim}(P(21,7)) \geq 4$ by an exhaustive search. Let $W=\left\{v_{14}, v_{17}, v_{20}, v_{21}\right\}$. The code of $v$ with respect to $W$ in $P(21,7)$ is presented in Table 15 showing that $\operatorname{dim}(P(21,7)) \leq 4$.

If $k \geq 9$, let $W=\left\{u_{1}, v_{k}, v_{(3 k-3) / 2}\right\}$. Then the codes of the outer vertices are $c_{W}\left(u_{i}\right)=\left(h_{1}(i), h_{2}(i), h_{3}(i)\right)$ and the codes of the inner vertices are $c_{W}\left(v_{i}\right)=\left(g_{1}(i), g_{2}(i), g_{3}(i)\right)$, where

$$
h_{1}(i)= \begin{cases}i-1, & 1 \leq i \leq \frac{k+5}{2} ; \\ k+4-i, & \frac{k+7}{2} \leq i \leq k+1 ; \\ i+2-k, & k+2 \leq i \leq \frac{3 k+1}{2} ; \\ 2 k+2-i, & \frac{3 k+3}{2} \leq i \leq 2 k+1 ; \\ i-2 k+2, & 2 k+2 \leq i \leq \frac{5 k-1}{2} ; \\ 3 k-i+1, & \frac{5 k+1}{2} \leq i \leq 3 k .\end{cases}
$$


TABLE 14: The $\operatorname{code} c_{W}(v)$ of $v$ with respect to $W=\left\{v_{4}, v_{10}, v_{13}\right\}$ in $P(15,5)$.

\begin{tabular}{lccccccccc}
\hline$v$ & $c_{W}(v)$ & $v$ & $c_{W}(v)$ & $v$ & $c_{W}(v)$ & $v$ & $c_{W}(v)$ & $v$ & $c_{W}(v)$ \\
\hline$u_{1}$ & $(4,3,4)$ & $u_{2}$ & $(3,4,3)$ & $u_{3}$ & $(2,4,2)$ & $u_{4}$ & $(1,3,3)$ & $u_{5}$ & $(2,2,4)$ \\
$u_{6}$ & $(3,3,4)$ & $u_{7}$ & $(4,4,3)$ & $u_{8}$ & $(3,3,2)$ & $u_{9}$ & $(2,2,3)$ & $u_{10}$ & $(3,1,4)$ \\
$u_{11}$ & $(4,2,3)$ & $u_{12}$ & $(4,3,2)$ & $u_{13}$ & $(3,4,1)$ & $u_{14}$ & $(2,3,2)$ & $u_{15}$ & $(3,2,3)$ \\
$v_{1}$ & $(5,4,5)$ & $v_{2}$ & $(4,5,4)$ & $v_{3}$ & $(3,5,1)$ & $v_{4}$ & $(0,4,4)$ & $v_{5}$ & $(3,1,5)$ \\
$v_{6}$ & $(4,4,5)$ & $v_{7}$ & $(5,5,4)$ & $v_{8}$ & $(4,4,1)$ & $v_{9}$ & $(1,3,4)$ & $v_{10}$ & $(4,0,5)$ \\
$v_{11}$ & $(5,3,4)$ & $v_{12}$ & $(5,4,3)$ & $v_{13}$ & $(4,5,0)$ & $v_{14}$ & $(1,4,3)$ & $v_{15}$ & $(4,1,4)$ \\
\hline
\end{tabular}

$$
\begin{aligned}
& g_{1}(i)= \begin{cases}i, & 1 \leq i \leq \frac{k+3}{2} ; \\
k+3-i, & \frac{k+5}{2} \leq i \leq k+1 ; \\
i+1-k, & k+2 \leq i \leq \frac{3 k+1}{2} ; \\
2 k+3-i, & \frac{3 k+3}{2} \leq i \leq 2 k+1 ; \\
i-2 k+1, & 2 k+2 \leq i \leq \frac{5 k+1}{2} ; \\
3 k-i+2, & \frac{5 k+3}{2} \leq i \leq 3 k .\end{cases} \\
& h_{2}(i)= \begin{cases}i+2, & 1 \leq i \leq \frac{k-1}{2} ; \\
k+1-i, & \frac{k+1}{2} \leq i \leq k ; \\
i+1-k, & k+1 \leq i \leq \frac{3 k+1}{2} ; \\
2 k+2-i, & \frac{3 k+3}{2} \leq i \leq 2 k ; \\
i-2 k+2, & 2 k+1 \leq i \leq \frac{5 k-1}{2} ; \\
3 k-i+2, & \frac{5 k+1}{2} \leq i \leq 3 k .\end{cases} \\
& g_{2}(i)= \begin{cases}0, & i=k ; \\
1, & i \in\{2 k, 3 k\} ; \\
i+3, & 1 \leq i \leq \frac{k-1}{2} ; \\
k+2-i, & \frac{k+1}{2} \leq i \leq k-1 ; \\
i+2-k, & k+1 \leq i \leq \frac{3 k+1}{2} ; \\
2 k+3-i, & \frac{3 k+3}{2} \leq i \leq 2 k-1 ; \\
i-2 k+3, & 2 k+1 \leq i \leq \frac{5 k-1}{2} ; \\
3 k-i+3, & \frac{5 k+1}{2} \leq i \leq 3 k-1 .\end{cases}
\end{aligned}
$$$$
h_{3}(i)= \begin{cases}\frac{k+1}{2}-i, & 1 \leq i \leq \frac{k-3}{2} ; \\ i-1, & \frac{k-1}{2} \leq i \leq k-2 ; \\ k-i+4, & k-1 \leq i \leq \frac{3 k-3}{2} ; \\ i-k-2, & \frac{3 k-1}{2} \leq i \leq 2 k-1 ; \\ 2 k-i+5, & 2 k \leq i \leq \frac{5 k-3}{2} ; \\ i-2 k-1, & \frac{5 k-1}{2} \leq i \leq 3 k-2 ; \\ 3 k-i+5, & 3 k-1 \leq i \leq 3 k .\end{cases}
$$$$
g_{3}(i)= \begin{cases}0, & i=\frac{3 k-3}{2} ; \\ 1, & i \in\left\{\frac{k-3}{2}, \frac{5 k-3}{2}\right\} \\ \frac{k+3}{2}-i, & 1 \leq i \leq \frac{k-5}{2} \\ i, & \frac{k-1}{2} \leq i \leq k-2 ; \\ k-i+5, & k-1 \leq i \leq \frac{3 k-5}{2} \\ i-k-1, & \frac{3 k-1}{2} \leq i \leq 2 k-1 ; \\ 2 k-i+6, & 2 k \leq i \leq \frac{5 k-5}{2} \\ i-2 k, & \frac{5 k-1}{2} \leq i \leq 3 k-2 \\ 3 k-i+6, & 3 k-1 \leq i \leq 3 k .\end{cases}
$$

It can be verified that there are no two vertices on the outer cycle with the same codes, and there are no two vertices in the inner cycle and outer cycle with the same codes. Moreover, no two vertices in the inner cycle have same codes. Hence, $W=\left\{u_{1}, v_{k}, v_{(3 k-3) / 2}\right\}$ is a resolving set of $P(3 k, k)$ for odd $k \geq 9$. This means that $\operatorname{dim}(P(3 k, k)) \leq 3$ for odd $k \geq 9$.

Now, we will show that $\operatorname{dim}(P(3 k, k)) \geq 3$. Note that for any $w \in U$, say $w=u_{i}$, we have $\left|N_{2}(w)\right|=6$ (see Figure 1 ) and for any $w \in V$, say $w=v_{i}$, we have $\left|N_{3}(w)\right|=8$ (see Figure 2). By Lemma 7, we have $\operatorname{dim}(P(3 k, k)) \geq 3$. 
TABLE 15: The $\operatorname{code} c_{W}(v)$ of $v$ with respect to $W=\left\{v_{14}, v_{17}, v_{20}, v_{21}\right\}$ in $P(21,7)$.

\begin{tabular}{lccccccccc}
\hline$v$ & $c_{W}(v)$ & $v$ & $c_{W}(v)$ & $v$ & $c_{W}(v)$ & $v$ & $c_{W}(v)$ & $v$ & $c_{W}(v)$ \\
\hline$u_{1}$ & $(3,4,3,2)$ & $u_{2}$ & $(4,3,4,3)$ & $u_{3}$ & $(5,2,5,4)$ & $u_{4}$ & $(5,3,4,5)$ & $u_{5}$ & $(4,4,3,4)$ \\
$u_{6}$ & $(3,5,2,3)$ & $u_{7}$ & $(2,5,3,2)$ & $u_{8}$ & $(3,4,4,3)$ & $u_{9}$ & $(4,3,5,4)$ & $u_{10}$ & $(5,2,5,5)$ \\
$u_{11}$ & $(4,3,4,5)$ & $u_{12}$ & $(3,4,3,4)$ & $u_{13}$ & $(2,5,2,3)$ & $u_{14}$ & $(1,4,3,2)$ & $u_{15}$ & $(2,3,4,3)$ \\
$u_{16}$ & $(3,2,5,4)$ & $u_{17}$ & $(4,1,4,5)$ & $u_{18}$ & $(5,2,3,4)$ & $u_{19}$ & $(4,3,2,3)$ & $u_{20}$ & $(3,4,1,2)$ \\
$u_{21}$ & $(2,5,2,1)$ & $v_{1}$ & $(4,5,4,3)$ & $v_{2}$ & $(5,4,5,4)$ & $v_{3}$ & $(6,1,6,5)$ & $v_{4}$ & $(6,4,5,6)$ \\
$v_{5}$ & $(5,5,4,5)$ & $v_{6}$ & $(4,6,1,4)$ & $v_{7}$ & $(1,6,4,1)$ & $v_{8}$ & $(4,5,5,4)$ & $v_{9}$ & $(5,4,6,5)$ \\
$v_{10}$ & $(6,1,6,6)$ & $v_{11}$ & $(5,4,5,6)$ & $v_{12}$ & $(4,5,4,5)$ & $v_{13}$ & $(3,6,1,4)$ & $v_{14}$ & $(0,5,4,1)$ \\
$v_{15}$ & $(3,4,5,4)$ & $v_{16}$ & $(4,3,6,5)$ & $v_{17}$ & $(5,0,5,6)$ & $v_{18}$ & $(6,3,4,5)$ & $v_{19}$ & $(5,4,3,4)$ \\
$v_{20}$ & $(4,5,0,3)$ & $v_{21}$ & $(1,6,3,0)$ & & & & & & \\
\hline
\end{tabular}

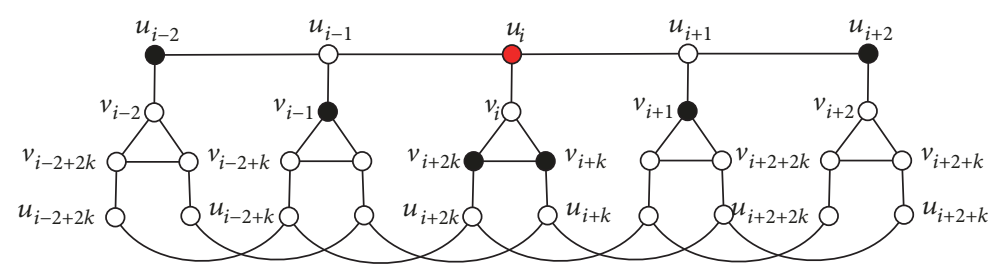

FIgURE 1: The set of black vertices is $N_{2}(w)$ for $w=u_{i}$.

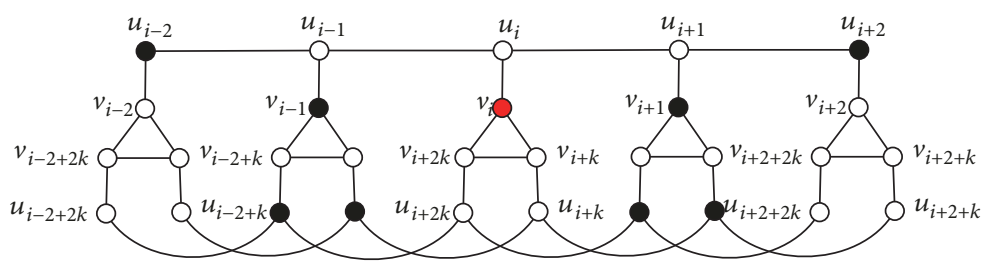

FIgURE 2: The set of black vertices is $N_{3}(w)$ for $w=v_{i}$.

\section{Data Availability}

The resolving sets of some generalized Petersen graphs can also be found in https://www.researchgate.net/publication/ 324182258_Resolving_sets_of_some_generalized_Petersen_ graphs_providing_the_corresponding_upper_bounds_for_ metric_dimension?.

\section{Disclosure}

The authors confirm that the paper has been read and approved by all named authors and that there are no other persons who satisfied the criteria for authorship but are not listed. The authors further confirm that the order of authors listed in the paper has been approved by all of them.

\section{Conflicts of Interest}

The authors wish to confirm that there are no known conflicts of interest associated with this paper and there has been no significant financial support for this work that could have influenced its outcome.

\section{Acknowledgments}

This work was supported by the National Key Research and Development Program under Grants 2017YFB0802300, the Key Project of the Sichuan Provincial Department of Education under Grants 17ZA0079 and 18ZA0118, the Soft Scientific Research Foundation of Sichuan Provincial Science and Technology Department under Grant 2018ZR0265, and Applied Basic Research (Key Project) of Sichuan Province under Grant 2017JY0095.

\section{References}

[1] F. Harary and R. A. Melter, "On the metric dimension of a graph," Ars Combinatoria, vol. 2, pp. 191-195, 1976.

[2] M. E. Watkins, "A theorem on Tait colorings with an application to the generalized Petersen graphs," Journal of Combinatorial Theory, vol. 6, pp. 152-164, 1969.

[3] P. S. Buczkowski, G. Chartrand, C. Poisson, and P. Zhang, "On k-dimensional graphs and their bases," Periodica Mathematica Hungarica, vol. 46, no. 1, pp. 9-15, 2003.

[4] R. Lang, T. Li, D. Mo, and Y. Shi, "A novel method for analyzing inverse problem of topological indices of graphs 
using competitive agglomeration," Applied Mathematics and Computation, vol. 291, pp. 115-121, 2016.

[5] S. Hayat, S. Wang, and J.-B. Liu, "Valency-based topological descriptors of chemical networks and their applications," Applied Mathematical Modelling: Simulation and Computation for Engineering and Environmental Systems, vol. 60, pp. 164-178, 2018.

[6] P. J. Slater, "Leaves of trees," Congressus Numerantium, vol. 14, pp. 549-559, 1975.

[7] S. Khuller, B. Raghavachari, and A. Rosenfeld, "Landmarks in graphs," Discrete Applied Mathematics: The Journal of Combinatorial Algorithms, Informatics and Computational Sciences, vol. 70, no. 3, pp. 217-229, 1996.

[8] M. Johnson, "Structure-activity maps for visualizing the graph variables arising in drug design," Journal of Biopharmaceutical Statistics, vol. 3, no. 2, pp. 203-236, 1993.

[9] M. Johnson, "Browsable structure-activity datasets," in Advances in Molecular Similarity Volume 2, vol. 2 of Advances in Molecular Similarity, pp. 153-170, Elsevier, 1999.

[10] R. F. Bailey and K. Meagher, "On the metric dimension of Grassmann graphs," Discrete Mathematics \& Theoretical Computer Science. DMTCS, vol. 13, no. 4, pp. 97-104, 2011.

[11] J. Cáceres, C. Hernando, M. Mora et al., "On the metric dimension of Cartesian products of graphs," SIAM Journal on Discrete Mathematics, vol. 21, no. 2, pp. 423-441, 2007.

[12] G. Chartrand, L. Eroh, M. A. Johnson, and O. R. Oellermann, "Resolvability in graphs and the metric dimension of a graph," Discrete Applied Mathematics, vol. 105, no. 1-3, pp. 99-113, 2000.

[13] M. Feng and K. Wang, "On the metric dimension of bilinear forms graphs," Discrete Mathematics, vol. 312, no. 6, pp. 12661268, 2012.

[14] Z. Shao, P. Wu, X. Zhang, D. Dimitrov, and J. Liu, "On the Maximum ABC Index of Graphs With Prescribed Size and Without Pendent Vertices," IEEE Access, vol. 6, pp. 2760427616, 2018.

[15] X. Zeng, X. Zhang, and Q. Zou, "Integrative approaches for predicting microRNA function and prioritizing disease-related microRNA using biological interaction networks," Briefings in Bioinformatics, vol. 17, no. 2, pp. 193-203, 2016.

[16] R. A. Melter and I. Tomescu, "Metric bases in digital geometry," Computer Vision, Graphics, and Image Processing, vol. 25, no. 1, pp. 113-121, 1984.

[17] V. Saenpholphat and P. Zhang, "Conditional resolvability in graphs: a survey," Int. J. Math. Math. Sci, vol. 38, 2004.

[18] H. M. Afzal Siddiqui and M. Imran, "Computing the metric dimension of wheel related graphs," Applied Mathematics and Computation, vol. 242, pp. 624-632, 2014.

[19] I. G. Yero, D. Kuziak, and J. A. Rodríguez-Velázquez, "On the metric dimension of corona product graphs," Computers \& Mathematics with Applications, vol. 61, no. 9, pp. 2793-2798, 2011.

[20] R. C. Brigham, G. Chartrand, R. D. Dutton, and P. Zhang, "Resolving domination in graphs," Academy of Sciences of the Czech Republic. Mathematical Institute. Mathematica Bohemica, vol. 128, no. 1, pp. 25-36, 2003.

[21] G. Chartrand, V. Saenpholphat, and P. Zhang, "The independent resolving number of a graph," Academy of Sciences of the Czech Republic. Mathematical Institute. Mathematica Bohemica, vol. 128, no. 4, pp. 379-393, 2003.

[22] F. Okamoto, B. Phinezy, and P. Zhang, "The local metric dimension of a graph," Academy of Sciences of the Czech Republic.
Mathematical Institute. Mathematica Bohemica, vol. 135, no. 3 , pp. 239-255, 2010.

[23] A. Sebo and E. Tannier, "On metric generators of graphs," Mathematics of Operations Research, vol. 29, no. 2, pp. 383-393, 2004.

[24] A. Kelenc, D. Kuziak, A. Taranenko, and I. G. Yero, "Mixed metric dimension of graphs," Applied Mathematics and Computation, vol. 314, pp. 429-438, 2017.

[25] I. G. Yero, A. Estrada-Moreno, and J. A. Rodríguez-Velázquez, "Computing the k-metric dimension of graphs," Applied Mathematics and Computation, vol. 300, pp. 60-69, 2017.

[26] L. Chen, Y. Ma, Y. Shi, and Y. Zhao, "On the [1,2]-domination number of generalized Petersen graphs," Applied Mathematics and Computation, vol. 327, pp. 1-7, 2018.

[27] I. Javaid, M. T. Rahim, and K. Ali, "Families of regular graphs with constant metric dimension," Utilitas Mathematica, vol. 75, pp. 21-33, 2008.

[28] C. Hernando, M. Mora, I. M. Pelayo, C. Seara, J. Cáceres, and M. L. Puertas, "On the metric dimension of some families of graphs," Electronic Notes in Discrete Mathematics, vol. 22, pp. 129-133, 2005.

[29] M. Imran, A. Q. Baig, M. K. Shafiq, and I. Tomecu, "On Metric Dimension of Generalized Petersen gpraphs $\mathrm{P}(\mathrm{n}, 3)$," Ars Combinatoria, vol. 117, pp. 113-130, 2014.

[30] S. Ahmad, M. A. Chaudhry, I. Javaid, and M. Salman, "On the metric dimension of generalized Petersen graphs," Quaestiones Mathematicae, vol. 36, no. 3, pp. 421-435, 2013.

[31] I. Javaid, S. Ahmad, and M. N. Azhar, "On the metric dimension of generalized Petersen graphs," Ars Combinatoria, vol. 105, pp. 171-182, 2012.

[32] S. Naz, M. Salman, U. Ali, I. Javaid, and S. A. Bokhary, "On the Constant Metric Dimension of Generalized Petersen Graphs P(n,4)," Acta Mathematica Sinica, vol. 30, no. 7, pp. 1145-1160, 2014. 


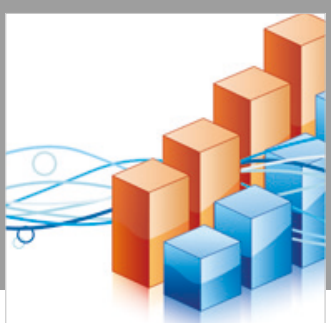

Advances in

Operations Research

\section{-n-m}
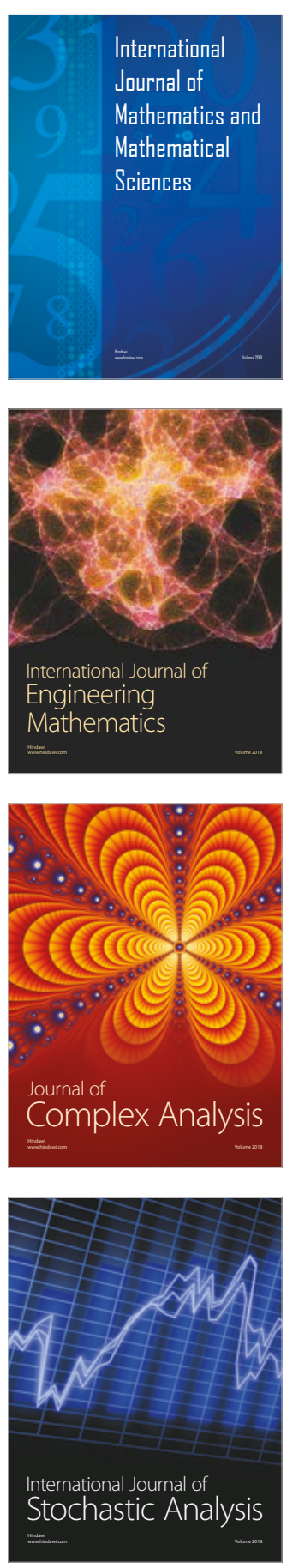
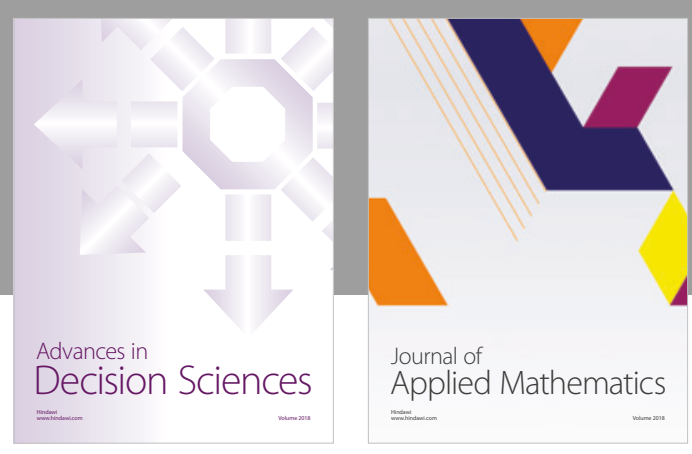

Journal of

Applied Mathematics
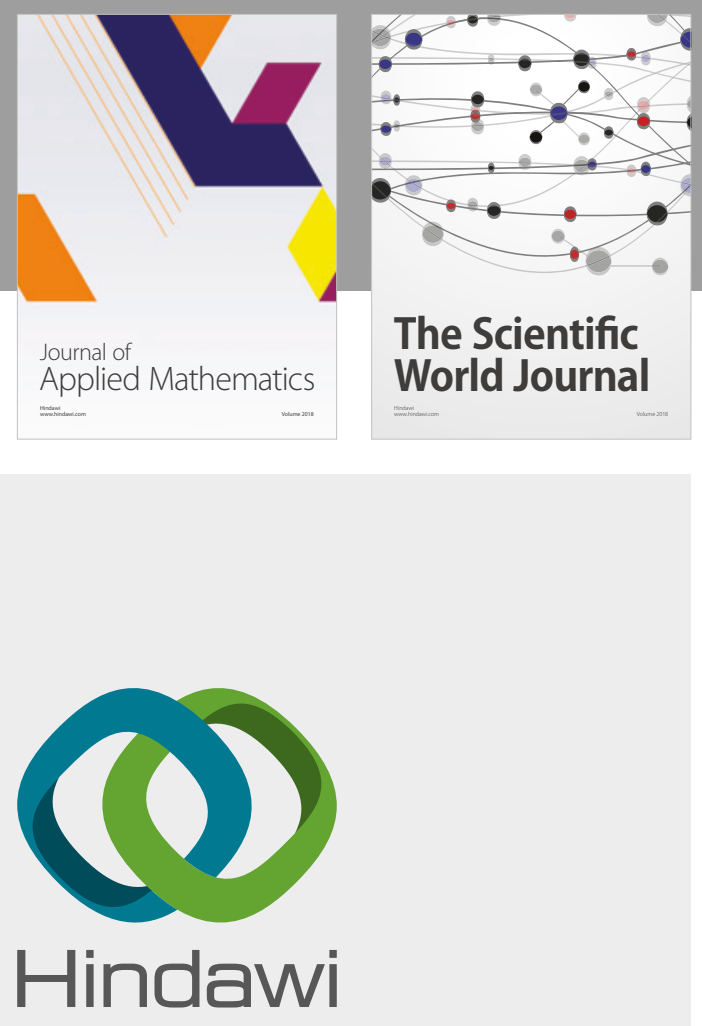

Submit your manuscripts at

www.hindawi.com

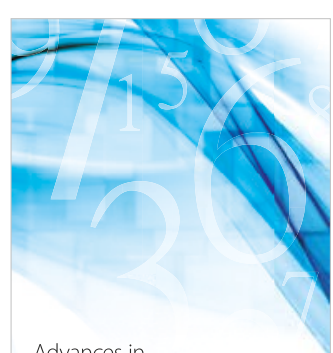

Advances in
Numerical Analysis
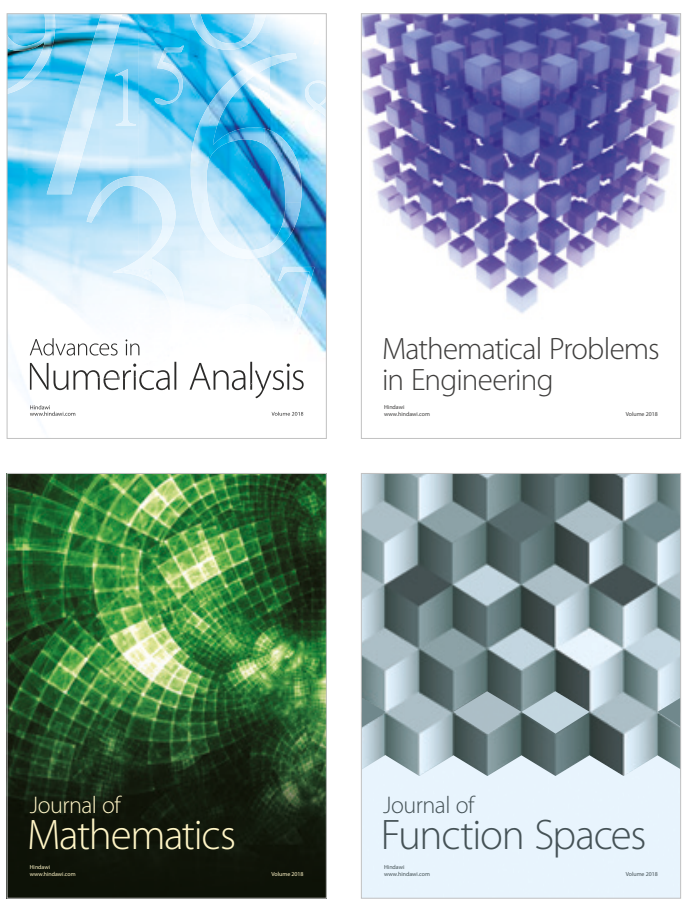

Mathematical Problems in Engineering

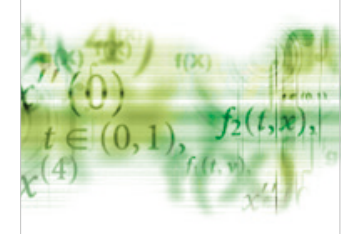

International Journal of

Differential Equations

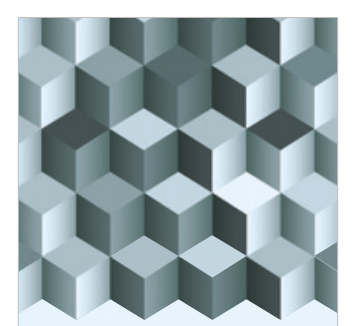

Journal of

Function Spaces

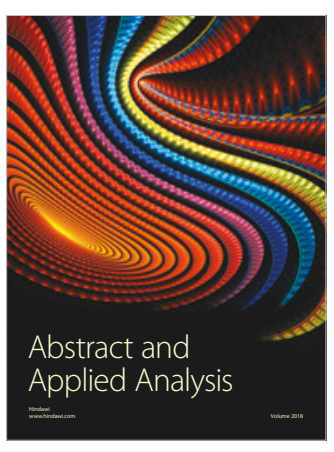

The Scientific

World Journal

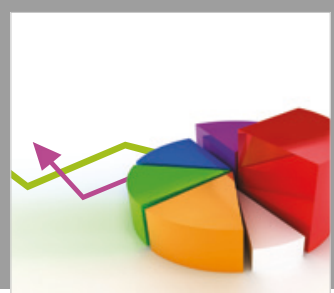

Journal of

Probability and Statistics
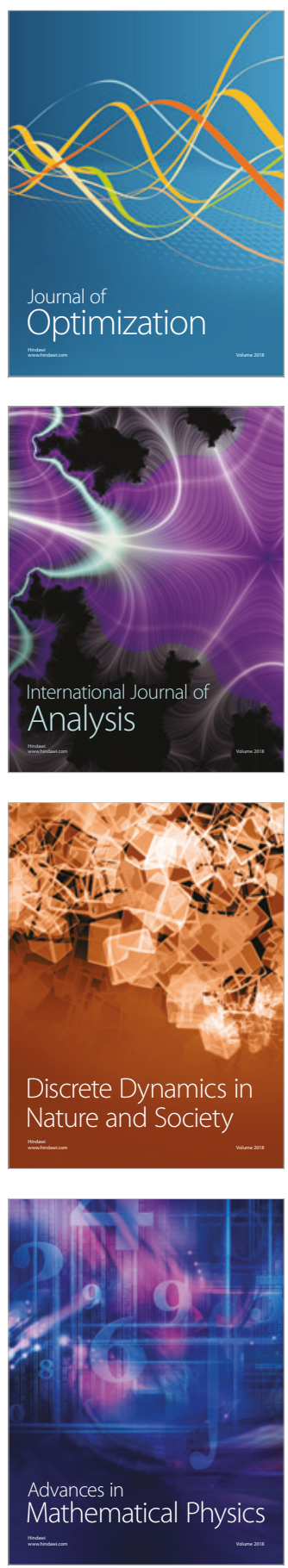